\title{
Output Feedback Variable Structure Control Design for Uncertain Nonlinear Lipschitz Systems
}

\author{
Jeang-Lin Chang and Tsui-Chou Wu \\ Department of Electrical Engineering, Oriental Institute of Technology, Banciao District, New Taipei City 220, Taiwan \\ Correspondence should be addressed to Jeang-Lin Chang; fe035@mail.oit.edu.tw
}

Received 18 June 2015; Revised 24 October 2015; Accepted 1 November 2015

Academic Editor: Ai-Guo Wu

Copyright ( 2015 J.-L. Chang and T.-C. Wu. This is an open access article distributed under the Creative Commons Attribution License, which permits unrestricted use, distribution, and reproduction in any medium, provided the original work is properly cited.

This paper develops a full-order compensator-based output feedback variable structure control law for uncertain nonlinear Lipschitz systems having matched perturbations. Given that the sufficient condition is satisfied, the developed control scheme, with the observer-like technique incorporated into the design of the compensator, can achieve global exponential stabilization. An illustrative example is provided with simulation results to show the effectiveness of the proposed method.

\section{Introduction}

The output feedback control problem for linear and/or nonlinear systems has received considerable attention in the literature due to its importance in many practical applications. There are two major classes of output feedback control schemes: static output feedback scheme and observerbased output feedback scheme. In observer-based output feedback control, an observer is first designed to estimate unmeasurable states, and the outputs as well as the estimated states are then used to design the control law. Static output feedback control, which involves either direct output feedback control or a dynamic compensator, does not use any observer to estimate the unmeasurable states. In recent years, many static output feedback sliding mode control (OFSMC) methods [1-9] have been proposed for stabilizing multivariable plants with matched perturbations. Edwards and Spurgeon [1] have developed a synthesizing output feedback controller according to sliding mode concepts. For the plant does not satisfy the Kimura-Davison conditions, different compensator design methods have been proposed [4-9] to provide additional degree of freedom, thus obviating this constraint. However, these above-mentioned methods [1-9] cannot be implemented in nonlinear systems.

Many nonlinear systems satisfy the Lipschitz property at least locally by representing them by a linear part with
Lipschitz nonlinearity around their equilibrium points. Hence, the systems with Lipschitz nonlinearities are common in practical applications. Prior studies [10-16] have developed different observer design methods for uncertain nonlinear Lipschitz systems. Nevertheless, separation principle does not generally hold for nonlinear systems. Therefore, the output feedback control problem for nonlinear Lipschitz systems is much more challenging than stabilization using full state feedback. In this paper, a solution to the output feedback variable structure control problem for uncertain nonlinear Lipschitz systems is provided. For the plant having matched perturbations and Lipschitz nonlinearity, an observer-like reference model design method is proposed and the existence condition proposed here is equivalent to the same condition of the sliding mode observer proposed by Koshkouei and Zinober [13]. Corless and $\mathrm{Tu}$ [17] presented a sufficient and necessary condition for the existence of this observer. Moreover, this problem can be taken as a convex optimization problem and LMI methods in prior studies $[13,18]$ can be employed to synthesize the parameters in the control algorithm. Note that the proposed method does not need any observer structure to estimate the system states and is a type of the dynamic compensator-based design method. Given that the condition of the controller problem is satisfied, the proposed control law can achieve global exponential stabilization. 


\section{Problem Formulations}

Consider a class of nonlinear systems described by the following equations [11-15]:

$$
\begin{aligned}
& \dot{\mathbf{x}}(t)=\mathbf{A} \mathbf{x}(t)+\mathbf{B}(\mathbf{u}(t)+\mathbf{d}(t))+\mathbf{f}(\mathbf{x}, \mathbf{u}), \\
& \mathbf{y}(t)=\mathbf{C} \mathbf{x}(t),
\end{aligned}
$$

where $\mathbf{x} \in \mathfrak{R}^{n}, \mathbf{u} \in \mathfrak{R}^{m}$, and $\mathbf{y} \in \mathfrak{R}^{l}$ are the system states, control inputs, and measureable outputs, respectively. The function $\mathbf{d} \in \mathfrak{R}^{m}$ is unknown and is a time-varying vector which represents the lump sum of matched disturbances and/or uncertainties. The function $\mathbf{f}: \mathfrak{R}^{n} \times \mathfrak{R}^{m} \rightarrow \mathfrak{R}^{n}$ is a nonlinear smooth vector with $\mathbf{f}(\mathbf{0}, \mathbf{0})=\mathbf{0}$ and satisfies the Lipschitz condition with respect to $\mathbf{x}$, with Lipschitz constant $\gamma>0$; that is, for all $\mathbf{x}_{1}$ and $\mathbf{x}_{2}$

$$
\left\|\mathbf{f}\left(\mathbf{x}_{1}, \mathbf{u}\right)-\mathbf{f}\left(\mathbf{x}_{2}, \mathbf{u}\right)\right\| \leq \gamma\left\|\mathbf{x}_{1}-\mathbf{x}_{2}\right\|,
$$

and $\|\mathbf{f}(\mathbf{x}, \mathbf{u})\| \leq \gamma\|\mathbf{x}\|$ for all $\mathbf{u} \in \mathfrak{R}^{m}$. In the proposed approach, the observer-like dynamic compensator is first employed to generate the reference states and the output feedback variable structure controller is then designed to stabilize system (1). Throughout this study, the following three assumptions, which were generally developed from conventional output feedback sliding mode control design methods [1-9], are made.

Assumption 1. The triple $(\mathbf{C}, \mathbf{A}, \mathbf{B})$ is minimum phase and $\operatorname{rank}(\mathbf{C B})=\operatorname{rank}(\mathbf{B})=m$.

Assumption 2. The pairs (A, B) and (A, C) are stabilizable and detectable, respectively.

Assumption 3. The matched perturbation is bounded by

$$
\|\mathbf{d}(t)\| \leq \alpha(t, \mathbf{y}),
$$

where the scalar-valued function $\alpha(t, \mathbf{y})>0$ is known.

\section{Output Feedback Variable Structure Controller Design}

Although system (1) is imposed on the unknown disturbance and Lipschitz nonlinearity, the control objective of this section is to design the output feedback variable structure controller that can achieve globally exponential stabilization of the closed-loop system. First, the closed-loop reference model is generated by

$$
\begin{aligned}
\dot{\mathbf{x}}_{m}(t)= & (\mathbf{A}-\mathbf{B K}) \mathbf{x}_{m}(t)+\mathbf{L}\left(\mathbf{y}(t)-\mathbf{y}_{m}(t)\right) \\
& +\mathbf{f}\left(\mathbf{x}_{m}, \mathbf{u}\right), \\
\mathbf{y}_{m}(t)= & \mathbf{C} \mathbf{x}_{m}(t),
\end{aligned}
$$

where $\mathbf{x}_{m} \in \mathfrak{R}^{n}$ are the reference states and $\mathbf{L} \in \mathfrak{R}^{n \times l}$ is the gain designed in the latter. Let $\beta_{c}>0, \varepsilon_{1}>0$, and $\eta_{c}>0$ be the positive scalars. The gain $\mathbf{K} \in \mathfrak{R}^{m \times n}$ is decided by

$$
\mathbf{K}=\frac{\beta_{c}}{2} \mathbf{B}^{T} \mathbf{P}_{c},
$$

where the positive definite matrix $\mathbf{P}_{c}>0$ satisfies the following algebraic Riccati inequality:

$$
\begin{aligned}
& \mathbf{A}^{T} \mathbf{P}_{c}+\mathbf{P}_{c} \mathbf{A}-\beta_{c} \mathbf{P}_{c} \mathbf{B} \mathbf{B}^{T} \mathbf{P}_{c}+\varepsilon_{1} \mathbf{P}_{c} \mathbf{P}_{c}+\left(\frac{\gamma^{2}}{\varepsilon_{1}}+\eta_{c}\right) \mathbf{I}_{n} \\
& \quad<\mathbf{0} .
\end{aligned}
$$

For the plant in (1), we design the controller as

$$
\mathbf{u}(t)=-\mathbf{K x}_{m}(t)+\mathbf{v}(t),
$$

where the input $\mathbf{v} \in \mathfrak{R}^{m}$ is designed using sliding mode control. Substituting the control input (7) into system (1) yields

$$
\begin{aligned}
& \dot{\mathbf{x}}(t)=(\mathbf{A}-\mathbf{B K}) \mathbf{x}(t)+\mathbf{B}(\mathbf{v}(t)+\mathbf{d}(t)) \\
& +\mathbf{B K}\left(\mathbf{x}(t)-\mathbf{x}_{m}(t)\right)+\mathbf{f}(\mathbf{x}, \mathbf{u}) \\
& =(\mathbf{A}-\mathbf{B K}) \mathbf{x}(t)+\mathbf{B}(\mathbf{v}(t)+\mathbf{d}(t))+\mathbf{B K} \mathbf{e}_{x}(t) \\
& +\mathbf{f}(\mathbf{x}, \mathbf{u}) \text {, }
\end{aligned}
$$

where $\mathbf{e}_{x}=\mathbf{x}-\mathbf{x}_{m}$ and $\mathbf{e}_{y}=\mathbf{y}-\mathbf{y}_{m}=\mathbf{C} \mathbf{e}_{x}$ are the error states and error outputs, respectively. Then from (4) and (9), one can yield

$$
\begin{aligned}
& \dot{\mathbf{e}}_{x}(t)=(\mathbf{A}-\mathbf{B K}) \mathbf{e}_{x}(t)+\mathbf{B}(\mathbf{v}(t)+\mathbf{d}(t))+\mathbf{B K} \mathbf{e}_{x}(t) \\
& -\mathbf{L C} \mathbf{e}_{x}(t)+\mathbf{f}(\mathbf{x}, \mathbf{u})-\mathbf{f}\left(\mathbf{x}_{m}, \mathbf{u}\right) \\
& =(\mathbf{A}-\mathbf{L C}) \mathbf{e}_{x}(t)+\mathbf{B}(\mathbf{v}(t)+\mathbf{d}(t))+\mathbf{g}(t),
\end{aligned}
$$

where $\mathbf{g}(t)=\mathbf{f}(\mathbf{x}, \mathbf{u})-\mathbf{f}\left(\mathbf{x}_{m}, \mathbf{u}\right)$. It follows from (2) that

$$
\|\mathbf{g}(t)\|=\left\|\mathbf{f}(\mathbf{x}, \mathbf{u})-\mathbf{f}\left(\mathbf{x}_{m}, \mathbf{u}\right)\right\| \leq \gamma\left\|\mathbf{e}_{x}(t)\right\| .
$$

Now we design the sliding surface as

$$
\mathbf{s}(t)=\mathbf{F e}_{y}(t),
$$

where the matrices $\mathbf{F} \in \mathfrak{R}^{m \times l}$ are decided by

$$
\mathbf{F C}=\mathbf{B}^{T} \mathbf{P}_{o}
$$

Furthermore, given any positive number $\varepsilon_{2}>0$, the positive definite matrix $\mathbf{P}_{o}>0$ and the gain $\mathbf{L} \in \Re^{n \times l}$ satisfy the following algebraic Riccati inequality:

$$
\begin{gathered}
(\mathbf{A}-\mathbf{L C})^{T} \mathbf{P}_{o}+\mathbf{P}_{o}(\mathbf{A}-\mathbf{L C})+\varepsilon_{2} \mathbf{P}_{o} \mathbf{P}_{o} \\
+\left(\frac{\gamma^{2}}{\varepsilon_{2}}+\eta_{o}\right) \mathbf{I}_{n}<\mathbf{0}
\end{gathered}
$$

If the solution to the coupled equations (12) and (13) can be found, Koshkouei and Zinober [13] have shown that the sliding mode observer can be successfully implemented to estimate the system states. Furthermore, previous studies $[17,19]$ have given that there exist the matrices $\mathbf{L}, \mathbf{P}$, and F to satisfy the coupled equations (12) and (13) if and only if Assumptions 1 to 2 hold. Moreover, the way of finding 
the matrices $\mathbf{L}, \mathbf{P}$, and $\mathbf{F}$ that satisfy (12) and (13) has also been discussed in detail. With the help of LMI, the standard LMI forms which can be straightforwardly solved to obtain the solution of the coupled equations (12) and (13) are given in $[13,18]$. To stabilize the closed-loop system and eliminate the matched perturbation, the control input $\mathbf{v}(t)$ is designed as

$$
\begin{aligned}
\mathbf{v}(t) & =-(\alpha(t, \mathbf{y})+\rho) \frac{\mathbf{s}(t)}{\|\mathbf{s}(t)\|} \\
& =-(\alpha(t, \mathbf{y})+\rho) \frac{\mathbf{F e}_{y}(t)}{\left\|\mathbf{F e}_{y}(t)\right\|},
\end{aligned}
$$

where $\rho>0$ is a design constant. Now consider the following Lyapunov function:

$$
W\left(\mathbf{e}_{x}, \mathbf{x}_{m}\right)=\mathbf{e}_{x}^{T}(t) \mathbf{P}_{o} \mathbf{e}_{x}(t)+\zeta \mathbf{x}_{m}^{T}(t) \mathbf{P}_{c} \mathbf{x}_{m}(t),
$$

where $\varsigma>0$ is a constant. Let $V(t)=\mathbf{e}_{x}^{T}(t) \mathbf{P}_{o} \mathbf{e}_{x}(t)$ and take its time derivative to obtain

$$
\begin{aligned}
\dot{V}(t)= & \mathbf{e}_{x}^{T}(t)\left((\mathbf{A}-\mathbf{L C})^{T} \mathbf{P}_{o}+\mathbf{P}_{o}(\mathbf{A}-\mathbf{L C})\right) \mathbf{e}_{x}(t) \\
& +2 \mathbf{e}_{x}^{T}(t) \mathbf{P}_{o} \mathbf{B}(\mathbf{v}(t)+\mathbf{d}(t))+2 \mathbf{e}_{x}^{T}(t) \mathbf{P}_{o} \mathbf{g}(t) \\
\leq & \mathbf{e}_{x}^{T}(t)\left((\mathbf{A}-\mathbf{L C})^{T} \mathbf{P}_{o}+\mathbf{P}_{o}(\mathbf{A}-\mathbf{L C})\right) \mathbf{e}_{x}(t) \\
& +2\|\mathbf{s}(t)\|\|\mathbf{d}(t)\|-2\|\mathbf{s}(t)\|(\alpha(t, \mathbf{y})+\rho) \\
& +2 \mathbf{e}_{x}^{T}(t) \mathbf{P}_{o} \mathbf{g}(t) \\
\leq & \mathbf{e}_{x}^{T}(t)\left((\mathbf{A}-\mathbf{L C})^{T} \mathbf{P}_{o}+\mathbf{P}_{o}(\mathbf{A}-\mathbf{L C})\right) \mathbf{e}_{x}(t) \\
& -2 \rho\|\mathbf{s}(t)\|+2\left\|\mathbf{P}_{o} \mathbf{e}_{x}(t)\right\|\|\mathbf{g}(t)\| .
\end{aligned}
$$

Since (2) holds, one can yield

$$
\begin{aligned}
2\left\|\mathbf{P}_{o} \mathbf{e}_{x}(t)\right\|\|\mathbf{g}(t)\| \leq & \varepsilon_{2} \mathbf{e}_{x}^{T}(t) \mathbf{P}_{o} \mathbf{P}_{o} \mathbf{e}_{x}(t) \\
& +\frac{1}{\varepsilon_{2}} \mathbf{g}^{T}(t) \mathbf{g}(t) \\
\leq & \varepsilon_{2} \mathbf{e}_{x}^{T}(t) \mathbf{P}_{o} \mathbf{P}_{o} \mathbf{e}_{x}(t) \\
& +\frac{\gamma^{2}}{\varepsilon_{2}} \mathbf{e}_{x}^{T}(t) \mathbf{e}_{x}(t) .
\end{aligned}
$$

Substituting this inequality into (16) and applying the inequality in (16) to it yield

$$
\begin{aligned}
& \dot{V}(t) \leq \mathbf{e}_{x}^{T}(t) \\
& \cdot\left((\mathbf{A}-\mathbf{L C})^{T} \mathbf{P}_{o}+\mathbf{P}_{o}(\mathbf{A}-\mathbf{L C})+\varepsilon_{2} \mathbf{P}_{o} \mathbf{P}_{o}+\frac{\gamma^{2}}{\varepsilon_{2}} \mathbf{I}_{n}\right) \\
& \cdot \mathbf{e}_{x}(t)-2 \rho\|\mathbf{s}(t)\| \leq-\eta_{o}\left\|\mathbf{e}_{x}(t)\right\|^{2}-2 \rho\|\mathbf{s}(t)\| \\
& \leq-\eta_{o}\left\|\mathbf{e}_{x}(t)\right\|^{2} .
\end{aligned}
$$

Then taking the time derivative of (15) and applying the above inequality to it give

$$
\begin{aligned}
\dot{W} & \left(\mathbf{e}_{x}, \mathbf{x}_{m}\right) \leq-\eta_{o}\left\|\mathbf{e}_{x}(t)\right\|^{2} \\
& +\varsigma\left(\mathbf{x}_{m}^{T}(t)\left(\mathbf{A}^{T} \mathbf{P}_{c}+\mathbf{P}_{c} \mathbf{A}-\beta \mathbf{P}_{c} \mathbf{B B}^{T} \mathbf{P}_{c}\right) \mathbf{x}_{m}(t)\right. \\
& \left.+2 \mathbf{x}_{m}^{T}(t) \mathbf{P}_{c} \mathbf{f}\left(t, \mathbf{x}_{m}, \mathbf{u}\right)+2 \mathbf{x}_{m}^{T}(t) \mathbf{P}_{c} \mathbf{L} \mathbf{C} \mathbf{e}_{x}(t)\right) \\
& \leq-\eta_{o}\left\|\mathbf{e}_{x}(t)\right\|^{2} \\
& +\varsigma\left(\mathbf{x}_{m}^{T}(t)\left(\mathbf{A}^{T} \mathbf{P}_{c}+\mathbf{P}_{c} \mathbf{A}-\beta \mathbf{P}_{c} \mathbf{B} \mathbf{B}^{T} \mathbf{P}_{c}\right) \mathbf{x}_{m}(t)\right. \\
& \left.+2 \gamma\left\|\mathbf{P}_{c} \mathbf{x}_{m}(t)\right\|\left\|\mathbf{x}_{m}(t)\right\|+2 \mathbf{x}_{m}^{T}(t) \mathbf{P}_{c} \mathbf{L} \mathbf{C}_{x}(t)\right) .
\end{aligned}
$$

From

$$
\begin{aligned}
& 2 \gamma\left\|\mathbf{P}_{c} \mathbf{x}_{m}(t)\right\|\left\|\mathbf{x}_{m}(t)\right\| \\
& \quad \leq \varepsilon_{1} \mathbf{x}_{m}^{T}(t) \mathbf{P}_{c} \mathbf{P}_{c} \mathbf{x}_{m}(t)+\frac{\gamma^{2}}{\varepsilon_{1}} \mathbf{x}_{m}^{T}(t) \mathbf{x}_{m}(t),
\end{aligned}
$$

it follows that

$$
\begin{aligned}
& \dot{W}\left(\mathbf{e}_{x}, \mathbf{x}_{m}\right) \leq \varsigma\left(\mathbf{x}_{m}^{T}(t)\right. \\
& \cdot\left(\mathbf{A}^{T} \mathbf{P}_{c}+\mathbf{P}_{c} \mathbf{A}-\beta \mathbf{P}_{c} \mathbf{B} \mathbf{B}^{T} \mathbf{P}_{c}+\varepsilon_{1} \mathbf{P}_{c} \mathbf{P}_{c}+\frac{\gamma^{2}}{\varepsilon_{1}} \mathbf{I}_{n}\right) \\
& \left.\cdot \mathbf{x}_{m}(t)+2 \kappa\left\|\mathbf{e}_{x}(t)\right\|\left\|\mathbf{x}_{m}(t)\right\|\right)-\eta_{o}\left\|\mathbf{e}_{x}(t)\right\|^{2} \\
& \leq-\eta_{o}\left\|\mathbf{e}_{x}(t)\right\|^{2}-\eta_{c} \varsigma\left\|\mathbf{x}_{m}(t)\right\|^{2}+2 \varsigma \kappa\left\|\mathbf{e}_{x}(t)\right\| \\
& \cdot\left\|\mathbf{x}_{m}(t)\right\|,
\end{aligned}
$$

where $\kappa=\left\|\mathbf{P}_{c}\right\|\|\mathbf{L C}\|$. Choosing $\varsigma=\eta_{c} \eta_{o} / \kappa^{2}$ results in

$$
\dot{W}\left(\mathbf{e}_{x}, \mathbf{x}_{m}\right) \leq-\frac{1}{2} \eta_{o}\left\|\mathbf{e}_{x}(t)\right\|^{2}-\frac{1}{2} \eta_{c} \varsigma\left\|\mathbf{x}_{m}(t)\right\|^{2} .
$$

Therefore, $\mathbf{e}_{x}$ and $\mathbf{x}_{m}$ converge exponentially to zero. Since $\mathbf{e}_{x}=\mathbf{x}-\mathbf{x}_{m}$, then

$$
\mathbf{x}(t) \longrightarrow \mathbf{0} \text { as } t \longrightarrow \infty .
$$

Remark 4. To obtain the similar effect of the Luenberger observer, in this paper the term $\mathbf{L C} \mathbf{e}_{x}=\mathbf{L} \mathbf{e}_{y}$ is introduced into the reference model. Lavretsky and Wise [20] have first proposed this technique and applied it in adaptive control. Hence, the reference states can be considered equivalent to the system states.

Remark 5. From (4), (10), and (14), we can rewrite the sliding surface and the controller as

$$
\begin{aligned}
\mathbf{s}(t)= & \mathbf{F y}(t)-\mathbf{F C} \mathbf{x}_{m}(t), \\
\mathbf{u}(t)= & -\mathbf{K} \mathbf{x}_{m}(t)-(\alpha(t, \mathbf{y})+\rho) \frac{\mathbf{s}(t)}{\|\mathbf{s}(t)\|}, \\
\dot{\mathbf{x}}_{m}(t)= & (\mathbf{A}-\mathbf{B K}) \mathbf{x}_{m}(t)+\mathbf{L}\left(\mathbf{y}(t)-\mathbf{y}_{m}(t)\right) \\
& +\mathbf{f}\left(\mathbf{x}_{m}, \mathbf{u}\right) .
\end{aligned}
$$


Hence, the proposed control algorithm does not use any observer structure and is a type of the full-order compensator-based output feedback controller.

Remark 6. When system (1) does not involve Lipschitz nonlinearity, different compensator design methods for output feedback sliding mode control have been proposed in the literature [5-9]. The design problem of the sliding surface is thus formulated as a new static output feedback problem for an augmented system. Since it is well known that the general static output feedback problem is not convex, no complete solution to this problem is known. In the proposed method, the existing well-built LMI-tools can be employed to straightforwardly solve the coupled equations (12) and (13). As a result, the advantage of the proposed method is that an analytic solution has been obtained which obviates the numerical complexities of the above-mentioned approaches [5-9].

Remark 7. The main problem of implementing controller (24) is that a phenomenon called chattering is generated due to the discontinuous function $\mathbf{s}(t) /\|\mathbf{s}(t)\|$. It can be considered the undesired chattering effect produced by the high switching action of the control input. As a result, the chattering becomes the main implementation problem of sliding mode control. Numerous techniques have been proposed to eliminate this phenomenon. One of the most common solutions to reduce the chattering is the continuous approximation techniques. However, the continuous approximation techniques have the trade-off relation between the control performance and the chattering migration. Another drawback with applying the continuous approximation techniques is the reduction of the control accuracy.

\section{Numerical Example}

Consider the train system, proposed by Aldeen and Sharma [15], in which the state-space form of the model including both matched disturbance and Lipschitz nonlinearity is given by

$$
\begin{aligned}
\dot{\mathbf{x}}(t)= & {\left[\begin{array}{cccc}
0 & 1 & 0 & 0 \\
\frac{-k}{M_{E}} & -\alpha g & \frac{k}{M_{E}} & 0 \\
0 & 0 & 0 & 1 \\
\frac{k}{M_{C}} & 0 & \frac{-k}{M_{C}} & -\alpha g
\end{array}\right] \mathbf{x}(t) } \\
& +\left[\begin{array}{c}
0 \\
\frac{1}{M_{E}} \\
0 \\
0
\end{array}\right](u(t)+d(t)) \\
& +\left[\begin{array}{c}
\frac{\rho}{M_{E}}\left(x_{2}+x_{4}\right)^{2} \\
0 \\
\frac{\rho}{M_{E}}\left(x_{2}+x_{4}\right)^{2}
\end{array}\right]
\end{aligned}
$$

$$
\mathbf{y}(t)=\left[\begin{array}{llll}
1 & 0 & 0 & 0 \\
0 & 1 & 0 & 0
\end{array}\right] \mathbf{x}(t)
$$

where $\mathbf{x}=\left[\begin{array}{llll}x_{1} & x_{2} & x_{3} & x_{4}\end{array}\right]^{T}$ and $d(t)=\cos (t)+\sin (\pi t)$ is set as the unknown disturbance. For simulation purpose, the parameter values, being the same as those reported in [15], are $M_{E}=10 \mathrm{Kg}, M_{C}=10 \mathrm{Kg}, k=4.87 \mathrm{~N} / \mathrm{s}$, $\alpha=0.5 \mathrm{~s} / \mathrm{m}, \rho=1 \mathrm{~N} / \mathrm{s}$, and $g=9.8 \mathrm{~m} / \mathrm{s}^{2}$. It is easy to obtain this train system which does not satisfy the Kimura-Davison conditions and contains stable invariant zeros at $\{-4.6924,-0.2076\}$. Applying the developed control algorithm, we can obtain the following matrices:

$$
\begin{aligned}
& \mathbf{K}=\left[\begin{array}{llll}
37.4080 & 22.6673 & 14.3300 & 3.1712
\end{array}\right], \\
& \mathbf{L}=\left[\begin{array}{cc}
10 & 0 \\
0 & 0.6212 \\
1.4169 & 0 \\
1.4169 & 0
\end{array}\right] \\
& \mathbf{F}=\left[\begin{array}{ll}
0 & 0.1
\end{array}\right], \\
& \mathbf{P}_{c}=\left[\begin{array}{llll}
1.4408 & 0.1496 & 0.5065 & 0.1240 \\
0.1496 & 0.0907 & 0.0573 & 0.0127 \\
0.5065 & 0.0573 & 2.6441 & 0.4962 \\
0.1240 & 0.0127 & 0.4962 & 0.2028
\end{array}\right] \text {, } \\
& \mathbf{P}_{o}=\left[\begin{array}{cccc}
0.05 & 0 & -0.0072 & -0.0010 \\
0 & 1 & 0 & 0 \\
-0.0072 & 0 & 2.7804 & -0.5102 \\
-0.0010 & 0 & -0.5102 & 0.2035
\end{array}\right] \text {. }
\end{aligned}
$$

Hence, the reference model is designed as

$$
\begin{aligned}
& \dot{\mathbf{x}}_{m}=\left[\begin{array}{cccc}
0 & 1 & 0 & 0 \\
-4.2278 & -7.1667 & -0.9460 & -0.3171 \\
0 & 0 & 0 & 1 \\
0.9740 & 0 & -0.9740 & -4.9000
\end{array}\right] \mathbf{x}_{m} \\
& +\left[\begin{array}{cc}
10 & 0 \\
0 & 0.6212 \\
1.4169 & 0 \\
1.4169 & 0
\end{array}\right]\left(\mathbf{y}-\mathbf{y}_{m}\right) \\
& +\left[\begin{array}{c}
0 \\
0.1\left(x_{m 2}+x_{m 4}\right)^{2} \\
0 \\
0.2\left(x_{m 2}+x_{m 4}\right)^{2}
\end{array}\right] \\
& \mathbf{y}_{m}=\left[\begin{array}{llll}
1 & 0 & 0 & 0 \\
0 & 1 & 0 & 0
\end{array}\right] \mathbf{x}_{m}
\end{aligned}
$$




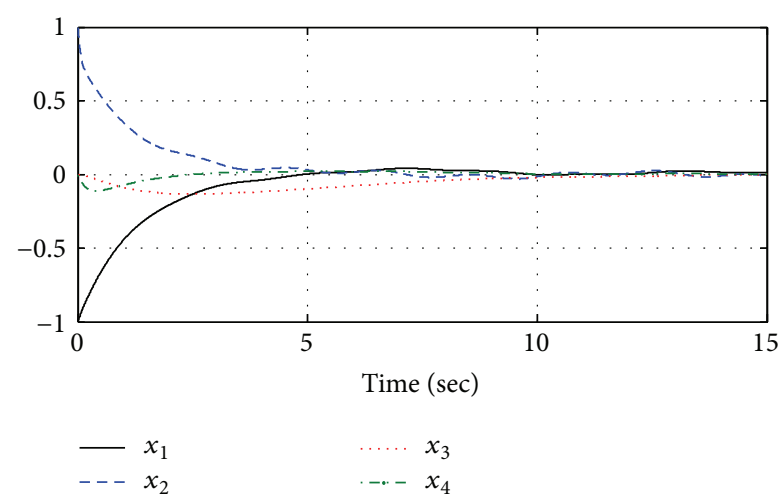

FIgURE 1: System states of the proposed method.

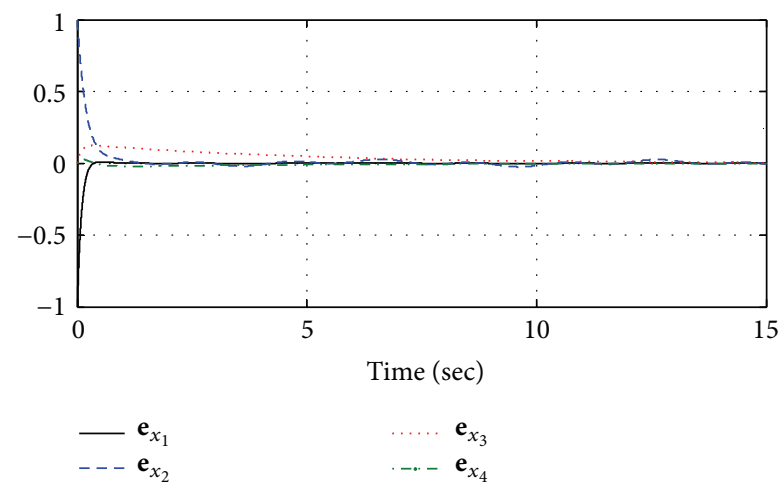

Figure 2: Error states of the proposed method.

and the sliding surface is chosen as

$$
\mathbf{s}(t)=\left[\begin{array}{ll}
0 & 0.1
\end{array}\right]\left(\mathbf{y}(t)-\mathbf{y}_{m}(t)\right) .
$$

To avoid the chattering problem, we apply the continuous approximation in the controller and then design the control inputs as

$$
\begin{aligned}
\mathbf{u}(t)= & -\left[\begin{array}{llll}
37.4080 & 22.6673 & 14.3300 & 3.1712
\end{array}\right] \mathbf{x}_{m}(t) \\
& -2 \frac{\mathbf{s}(t)}{\|\mathbf{s}(t)\|+0.01} .
\end{aligned}
$$

Figures 1-4 illustrate the simulation results obtained using the initial conditions $\mathbf{x}(0)=\left[\begin{array}{llll}-1 & 1 & 0 & 0\end{array}\right]^{T}$. Figures 1 and 2 plot the evolution of the system states and error states, respectively. Figure 3 depicts the control input. The response of the sliding surface is given in Figure 4. As seen in Figure 1, although the nominal system has both matched disturbance and Lipschitz nonlinearity, the proposed control law can successfully restrain the effect of matched disturbance and achieve the desired performance. Moreover, robust stability of the closed-loop system can be guaranteed.

\section{Conclusions}

This study examines the output feedback variable structure control problem for a class of Lipschitz nonlinear systems

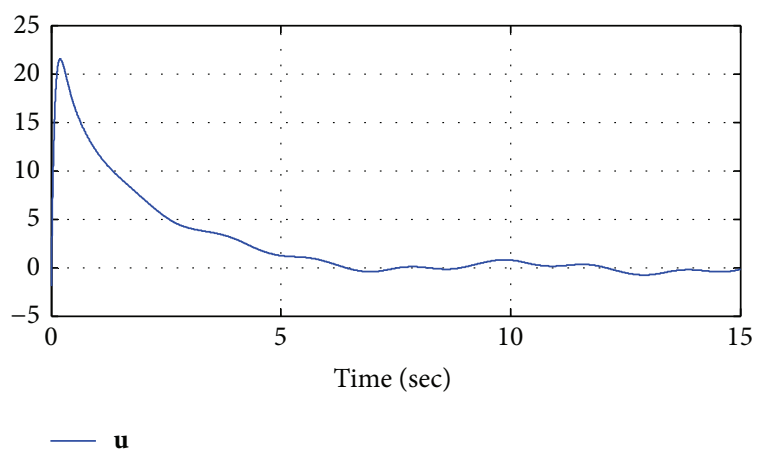

FIGURE 3: Response of the control input.

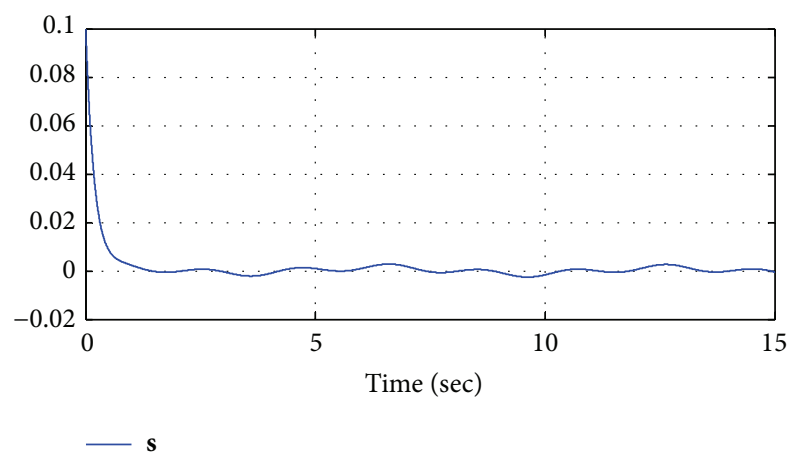

FIgURE 4: Response of the sliding surface.

with matched perturbations. The proposed method, which is simple by nature involving only its original system matrices and does not need any observer structure, can obtain exponential stabilization of the closed-loop system. The existence condition, which is equivalent to that used in sliding mode observer, is developed for the design of the proposed dynamic compensator-based controller. The numerical example demonstrates that the proposed algorithm can be successfully implemented.

\section{Conflict of Interests}

The authors declare that there is no conflict of interests regarding the publication of this paper.

\section{References}

[1] C. Edwards and S. K. Spurgeon, Sliding Mode Control Theory and Application, Taylor \& Francis, London, UK, 1998.

[2] R. El-Khazali and R. Decarlo, "Output feedback variable structure control design," Automatica, vol. 31, no. 6, pp. 805-816, 1995.

[3] H. H. Choi, "Variable structure output feedback control design for a class of uncertain dynamic systems," Automatica, vol. 38, no. 2, pp. 335-341, 2002.

[4] J.-L. Chang, "Dynamic output integral sliding-mode control with disturbance attenuation," IEEE Transactions on Automatic Control, vol. 54, no. 11, pp. 2653-2658, 2009.

[5] S. K. Bag, S. K. Spurgeon, and C. Edwards, "Output feedback sliding mode design for linear uncertain systems," IEE 
Proceedings-Control Theory and Applications, vol. 144, no. 3, pp. 209-216, 1997.

[6] C. Edwards and S. K. Spurgeon, "Compensator based output feedback sliding mode controller design," International Journal of Control, vol. 71, no. 4, pp. 601-614, 1998.

[7] C. Edwards and S. K. Spurgeon, "Linear matrix inequality methods for designing output feedback sliding mode controllers," IEE Proceedings-Control Theory and Applications, vol. 203, pp. 539-545, 2003.

[8] C. Edwards, S. K. Spurgeon, and R. G. Hebden, "On the design of sliding mode output feedback controller," International Journal of Control, vol. 76, no. 9-10, pp. 893-905, 2003.

[9] H. H. Choi, "Sliding-mode output feedback control design," IEEE Transactions on Industrial Electronics, vol. 55, no. 11, pp. 4047-4054, 2008.

[10] R. Rajamani, “Observers for Lipschitz nonlinear systems," IEEE Transactions on Automatic Control, vol. 43, no. 3, pp. 397-401, 1998.

[11] A. M. Pertew, H. J. Marquez, and Q. Zhao, " $H_{\infty}$ synthesis of unknown input observers for non-linear Lipschitz systems," International Journal of Control, vol. 78, no. 15, pp. 1155-1165, 2005.

[12] Q. P. Ha and H. Trinh, "State and input simultaneous estimation for a class of nonlinear systems," Automatica, vol. 40, no. 10, pp. 1779-1785, 2004.

[13] A. J. Koshkouei and A. S. I. Zinober, "Sliding mode state observation for non-linear systems," International Journal of Control, vol. 77, no. 2, pp. 118-127, 2004.

[14] P. R. Pagilla and Y. Zhu, "Controller and observer design for Lipschitz nonlinear systems," in Proceedings of the American Control Conference (AAC '04), pp. 2379-2384, IEEE, Boston, Mass, USA, July 2004.

[15] M. Aldeen and R. Sharma, "Estimation of states, faults and unknown disturbances in non-linear systems," International Journal of Control, vol. 81, no. 8, pp. 1195-1201, 2008.

[16] H.-L. Choi and J.-T. Lim, “Output feedback stabilization for a class of Lipschitz nonlinear systems," IEICE Transactions on Fundamentals of Electronics, Communications and Computer Sciences, vol. 88, no. 2, pp. 602-605, 2005.

[17] M. Corless and J. Tu, "State and input estimation for a class of uncertain systems," Automatica, vol. 34, no. 6, pp. 757-764, 1998.

[18] C. P. Tan and C. Edwards, "An LMI approach for designing sliding mode observers," International Journal of Control, vol. 74, no. 16, pp. 1559-1568, 2001.

[19] C. Edwards, X.-G. Yan, and S. K. Spurgeon, "On the solvability of the constrained Lyapunov problem," IEEE Transactions on Automatic Control, vol. 52, no. 10, pp. 1982-1987, 2007.

[20] E. Lavretsky and K. Wise, Robust and Adpative Control: With Aerospace Applications, Springer, Berlin, Germany, 2012. 

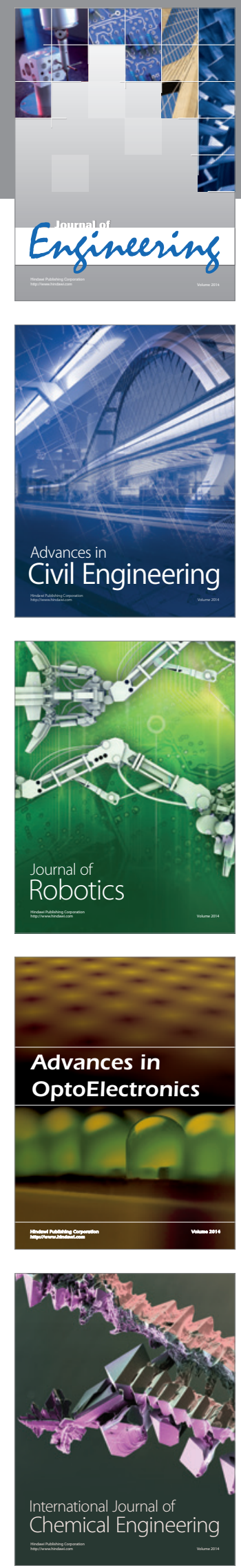

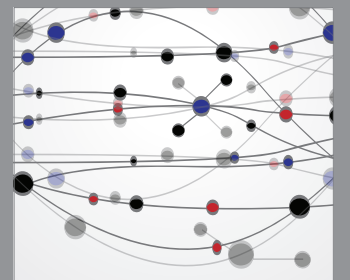

The Scientific World Journal
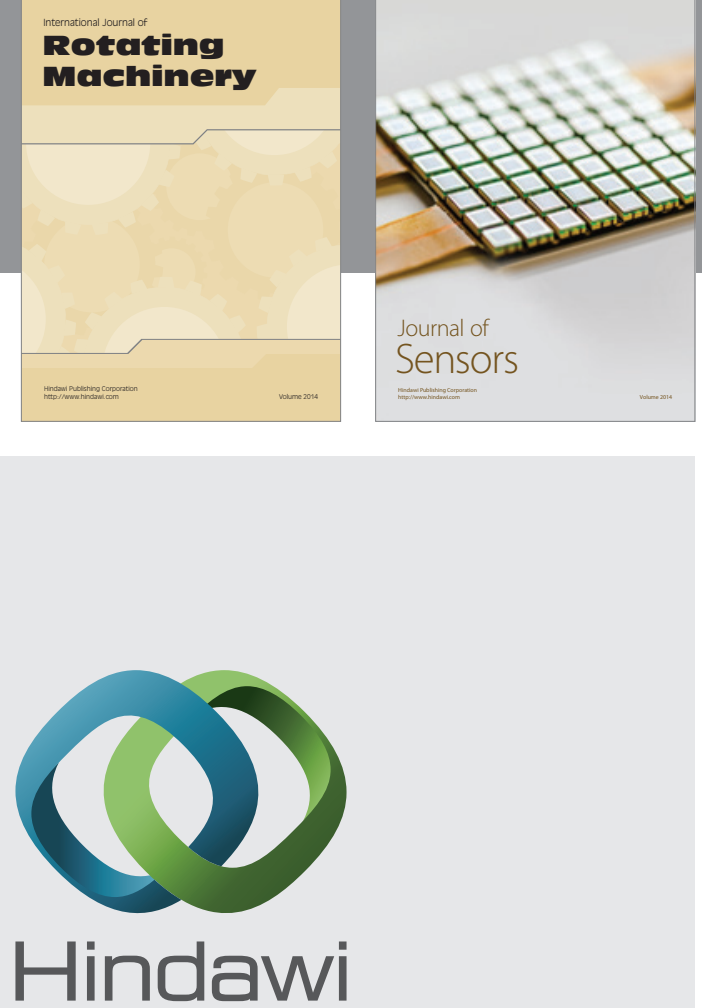

Submit your manuscripts at http://www.hindawi.com
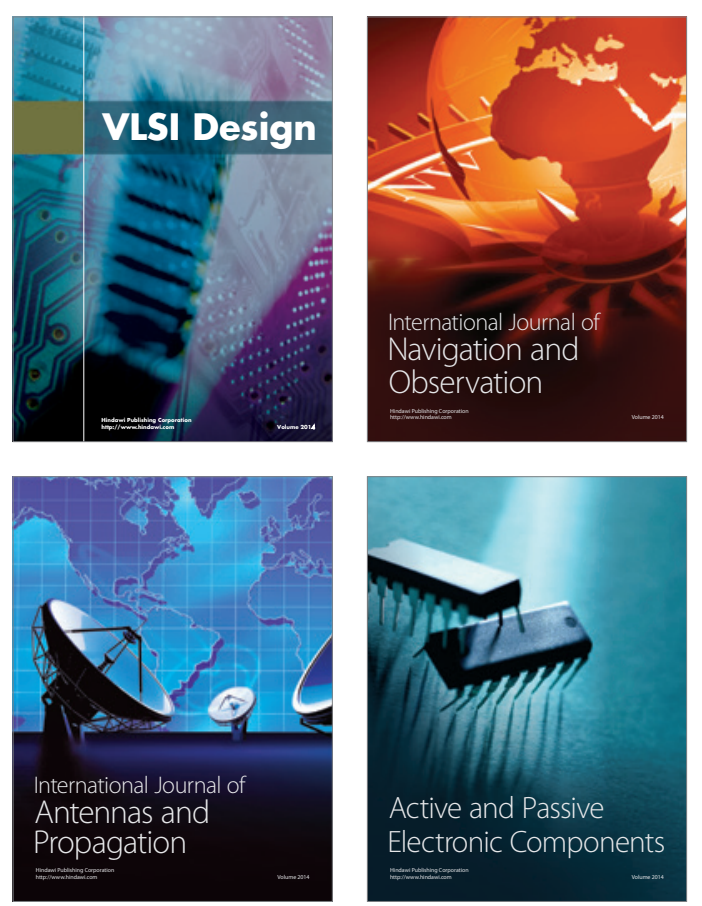
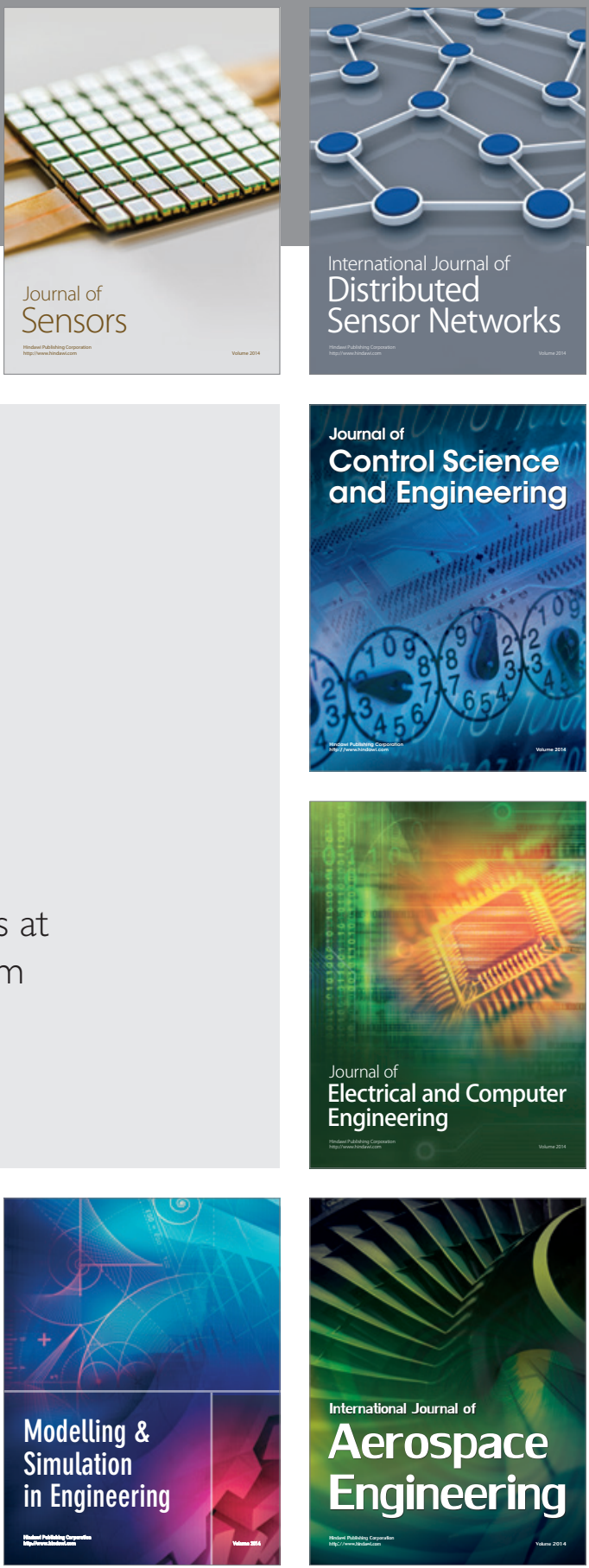

Journal of

Control Science

and Engineering
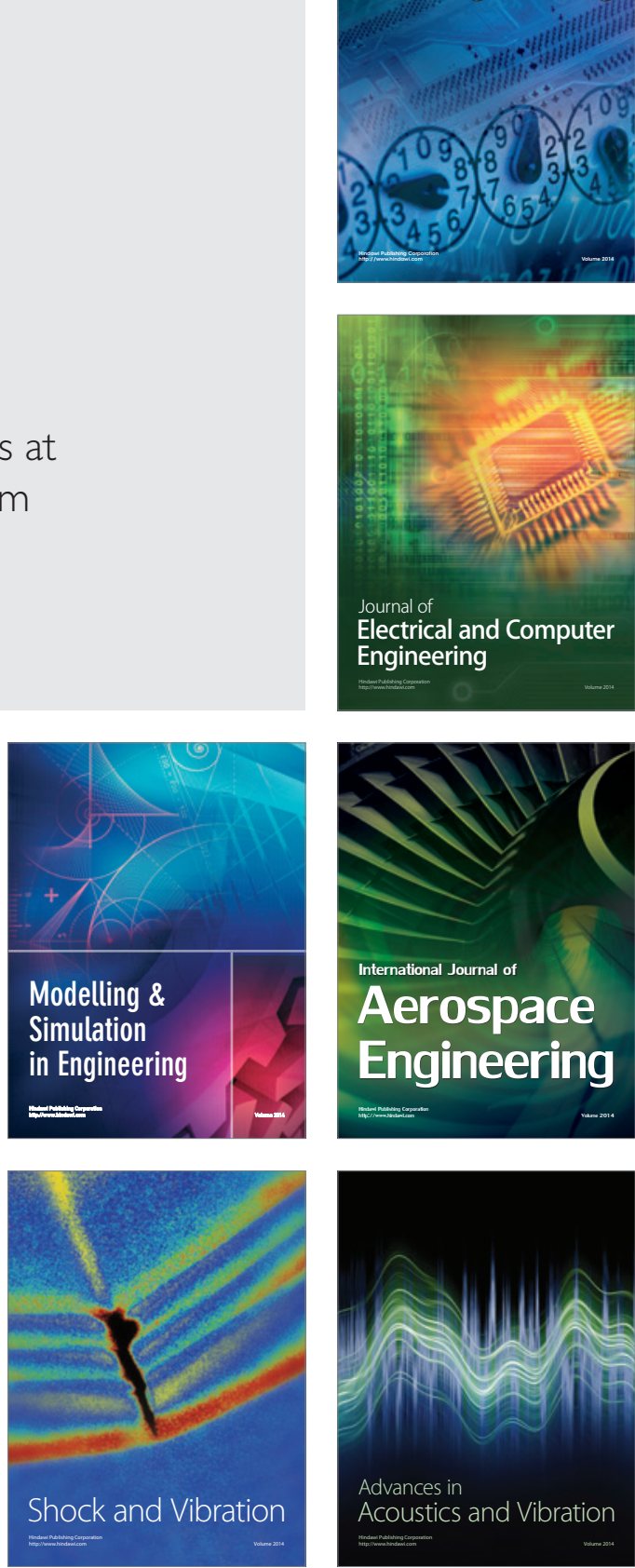